\title{
The application of capillary electrophoresis, mass spectrometry and Brdicka reaction in human and rabbit metallothioneins analysis
}

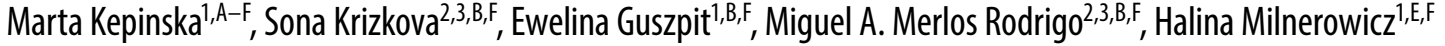 \\ ${ }^{1}$ Department of Biomedical and Environmental Analysis, Faculty of Pharmacy with Division of Laboratory Medicine, Wroclaw Medical University, Poland \\ 2 Department of Chemistry and Biochemistry, Faculty of Agronomy, Mendel University in Brno, Czech Republic \\ ${ }^{3}$ Central European Institute of Technology, Brno University of Technology, Czech Republic \\ A - research concept and design; B - collection and/or assembly of data; C - data analysis and interpretation; \\ $D$ - writing the article; $E$ - critical revision of the article; $F$ - final approval of the article
}

\section{Address for correspondence}

Marta Kepinska

E-mail:zalewska.m@gmail.com

Funding sources

Study was supported by grant No. Pbmn178

from Wroclaw Medical University and by the AZV

(Czech Agency for Healthcare Research) AZV CR

grant No. 14-28334a (Prague, Czech Republic).

Conflict of interest

None declared

Received on June 6, 2018

Reviewed on August 3, 2018

Accepted on October 11, 2018
DOI

10.17219/acem/98916

Copyright

Copyright by Author(s)

This is an article distributed under the terms of the

Creative Commons Attribution Non-Commercial License

(http://creativecommons.org/licenses/by-nc-nd/4.0/)

\begin{abstract}
Background. Metallothioneins (MTs) constitute a family of evolutionary conserved low molecular weight proteins with small variations in their amino acid sequences. They play a role in the regulation of trace metals metabolism, in the detoxification of heavy metal ions and in mechanisms controlling growth, differentiation and proliferation of cells.

Objectives. The aim of this study was to evaluate the human and rabbit MTs purity and characterization using advanced analytical approaches. Due to the common use of MT from rabbit liver as a model protein, the properties of the rabbit and human MTs were compared.

Material and methods. Capillary electrophoresis (CE), matrix-assisted laser desorption and ionization time-of-flight mass spectrometry (MALDI-TOF-MS) and Brdicka reaction were used for human and rabbit MTs characterization.

Results. In chip CE analysis, changes in the range of 5-8 kDa corresponding to the MT monomer, as well as some peaks of 13-14 kDa corresponding to dimers in both species, were observed. Using MALDI-MS, rabbit (MT-2D) and human (MT-1A, MT-1G, MT-1G + Cd and MT-2A) MTs were identified. In the Brdicka reaction analysis, a lower concentration of MTs from both organisms coincided with a decrease in the signal corresponding to MT level (Cat2). However, human MT gave higher Cat2 peak than the same concentration $(0.025 \mathrm{mg} / \mathrm{mL})$ of rabbit MT.

Conclusions. The applied methods allowed for the characterization of MTs and gave complementary information about MT isoforms. Altered electrochemical activity of human and rabbit MTs, despite the same number of -sulfhydryl (-SH) groups, was observed, which may be due to different availability of MT cysteinyl groups.
\end{abstract}

Key words: metallothionein, mass spectrometry, capillary electrophoresis, Brdicka reaction 


\section{Introduction}

Metallothioneins (MTs) make up a large family of evolutionarily conserved low molecular weight, thermostable proteins, found in practically all life forms: in vertebrates, invertebrates, fungi, and even in plants. Their sizes range from 25 (fungal) to 84 (plant) amino acids. ${ }^{1} \mathrm{~A}$ single polypeptide chain of mammalian MT is composed of 61-68 mostly non-aromatic amino acids and contains 20 cysteinyl residues in highly conserved locations along the peptide chain. High number of cysteines in MTs explains their high capacity for binding metal ions through sulfhydryl groups, forming metal-thiolate complexes. Thus, their main proposed functions were derived from their role in the homeostatic control of a number of essential metals (copper $-\mathrm{Cu}$, zinc $-\mathrm{Zn}$ ), in detoxification of toxic ones (e.g., cadmium - Cd, mercury - $\mathrm{Hg}$ ), and in protective actions against oxidative stress conditions. ${ }^{2-4}$ This known fact forms the basis of MTs being used as possible biomarkers for metal exposure. ${ }^{5}$

Four isoforms of mammalian MT (MT-1, MT-2, MT3 , and MT-4) have been identified. Isoforms with minor differences, such as amino acid residue, have been detected as subgroups of the 2 major isoforms (MT- 1 and MT-2) and are termed subisoforms. These 2 main groups of MT isoforms have been classified according to human MT elution order by anionic-exchange chromatography: MT-1 and MT-2. ${ }^{6}$ They are the most widely distributed MT isoforms, expressed in many cell types in different tissues and organs. They can be induced by the administration of metals such as $\mathrm{Cd}, \mathrm{Zn}$ and $\mathrm{Cu}$; by oxidative stress; by glucocorticoids; and by cytokines. ${ }^{7}$ MT-1 and MT-2 are present in nearly all tissues and have been isolated from organs such as the liver, kidney and brain. MT-3 is expressed mostly in nervous tissue ${ }^{8}$ and MT- 4 has been detected in epithelial cells. ${ }^{9}$ Since various subisoforms are biosynthesized differentially, they could play a specific biological role, but this aspect is still being investigated. The interesting properties of MTs are closely related to their structure. In addition to slight differences in amino acid sequences, MTs exist as a mixture of varying metallic content. ${ }^{5}$ It is not a simple task to detect and quantify MT, also due to its high cysteine content and relatively low molecular mass. The characteristics of human MTs are not widely described because rabbit MTs are more often used in studies as a model protein; thus, it is so valuable to compare MTs from these organisms. To analyze MTs, many methods can be used that differ in the analytical approach, usually based on the metal ions detection, free thiol moieties or enzyme-linked immunosorbent assay (ELISA). Human and rabbit MTs have previously been analyzed by western blot, ELISA, capillary electrophoresis (CE), and high-performance liquid chromatography. ${ }^{10-15}$

Therefore, the purpose of the research was the comprehensive characterization of human and rabbit MTs. Modern electrochemical and advanced analytical approaches such as the Brdicka reaction, chip CE and matrix-assisted laser desorption and ionization time-of-flight mass spectrometry (MALDI-TOF-MS) were used to characterize and compare human and rabbit MTs.

\section{Material and methods}

\section{Material}

All chemicals used were commercially available. Rabbit liver MT-2 (M-5392), 2-mercaptoethanol, ammonia solution, ammonium bicarbonate, trifluoroacetic acid (TFA), and formic acid were purchased from Sigma-Aldrich (St. Louis, USA). Acetonitrile (ACN) and methanol were purchased from Fluka (Seelze, Germany). Acidic MALDI matrices $\alpha$-cyano4-hydroxycinnamic acid (HCCA), 2,5-dihydroxobenzoic acid (DHB) and peptide calibration standard mixture were purchased from Bruker Daltonics (Bremen, Germany). All reagents were of the highest available purity. Doubly distilled deionized water was used throughout all the experiments.

\section{Preparation of metallothioneins from human liver}

Isoforms MT-1 and MT-2 were purified from human liver earlier at the Department of Biomedical and Environmental Analysis, Faculty of Pharmacy with Division of Laboratory Medicine, Wroclaw Medical University, Poland, and the presence of MTs was confirmed by sodium dodecyl sulfate-polyacrylamide gel electrophoresis (SDSPAGE) and western blot. ${ }^{10}$

\section{Chip capillary electrophoresis}

Capillary electrophoresis experiments were performed on an automated microfluidic Experion electrophoresis system (Bio-Rad, Hercules, USA), according to the manufacturer's instructions and with supplied chemicals (Experion Pro260 analysis kit, Cat. No. 700-7101, Bio-Rad). Metallothioneins in a quantity of $4 \mu \mathrm{L}(0.1$ or $0.18 \mu \mathrm{g} / \mu \mathrm{l})$ were mixed with $2 \mu \mathrm{L}$ of reducing sample buffer $(30 \mu \mathrm{L}$ of a nonreducing sample buffer and $1 \mu \mathrm{L}$ of $\beta$-mercaptoethanol); after 4 min of boiling, $84 \mu \mathrm{L}$ of water were added. The ladder was also prepared in reducing conditions. After the priming of the chip with the gel and gel-staining solution in the diluted priming station sample, $6 \mu \mathrm{L}$ of the mixture were loaded into the sample wells. The Pro260 Ladder included in the kit was used as a standard. For operation and standard data analysis, Experion software v. 3.10 (Bio-Rad) was used.

\section{Mass spectrometry analysis}

Mass spectrometry analysis was performed using matrix-assisted laser desorption and ionization time-of-flight mass spectrometry (MALDI-TOF-MS). Working standard 
solutions were prepared daily by diluting the stock solutions. A mixture of peptide calibration standard (Bruker Daltonics) was used to calibrate the instrument. All measurements were performed on the UltrafleXtreme MALDITOF/TOF mass spectrometer (Bruker Daltonics) equipped with a laser operating at a wavelength of $355 \mathrm{~nm}$ with an accelerating voltage of $25 \mathrm{kV}$, cooled with liquid nitrogen, at a maximum energy of $36 \mu \mathrm{J}$ with a repetition rate of $2000 \mathrm{~Hz}$ in reflector-positive ionization mode, and with software for data acquisition and processing of mass spectra flexControl v. 3.4 and flexAnalysis v. 2.2. The spectra were measured in the range of 0-20,000 mass-to-change ratio $(\mathrm{m} / \mathrm{z})$. Laser power intensity was set to $5 \%$ above the threshold, which determines the minimal intensity for suitable spectrum; it is set experimentally by measuring spectra with increasing laser power intensity. A MALDI MTP 384 target plate (polished steel) was used.

Before MT analysis, a cut-off filter (Amicon 3K; Merck Millipore, Burlington, USA) for desalinization of the MT sample and buffer exchange to water was used. Then, the samples were mixed with DHB or HCCA matrix solution in $1: 1$ volume ratio and then $1 \mu \mathrm{L}$ of the mixture was deposited on the target plate (dried-droplet method) and dried under atmospheric pressure and ambient temperature. Saturated solutions of DHB or HCCA were prepared in TA30 (30\% ACN and 0.1\% TFA). Mass spectra were typically acquired by averaging 20 subspectra from a total of 500 shots of the laser (Smartbeam 2 v. 1_0_38.5; Bruker Daltonics).

\section{Differential pulse voltammetry}

Differential pulse voltammetry (DPV) measurements were performed with a 747 VA Stand instrument connected to a 746 VA Trace Analyzer and a 695 Autosampler (Metrohm, Herisau, Switzerland) using a standard cell with 3 electrodes and a cooled sample holder $\left(4^{\circ} \mathrm{C}\right)$. A hanging mercury drop electrode (HMDE) with a drop area of $0.4 \mathrm{~mm}^{2}$ was used as the working electrode. An $\mathrm{Ag} / \mathrm{AgCl} / 3 \mathrm{M} \mathrm{KCl}$ was the reference electrode and a glassy carbon electrode was the auxiliary electrode. The GPES v. 4.9 software supplied by EcoChemie (Utrecht, the Netherlands) was employed for smoothing and baseline correction of the raw data. The Brdicka supporting electrolyte containing $1 \mathrm{mM} \mathrm{Co}\left(\mathrm{NH}_{3}\right)_{6} \mathrm{Cl}_{3}$ and $1 \mathrm{M}$ ammonia buffer $\left(\mathrm{NH}_{3}(\mathrm{aq})+\mathrm{NH}_{4} \mathrm{Cl}, \mathrm{pH}=9.6\right)$ was used. The parameters of the measurement were as follows: initial potential of $-0.7 \mathrm{~V}$, end potential of $-1.75 \mathrm{~V}$, modulation time $0.057 \mathrm{~s}$, time interval $0.2 \mathrm{~s}$, step potential $2 \mathrm{mV}$, and modulation amplitude $-250 \mathrm{mV}$. All experiments were carried out at $4^{\circ} \mathrm{C}$ employing a Julabo F12 thermostat (Julabo Labortechnik $\mathrm{GmbH}$, Seelbach, Germany).

\section{Results and discussion}

\section{Sequence differences in human and rabbit metallothioneins}

Most studies concerning mammalian MTs usually associate MT-1 with MT-2. These 2 isoforms are examined as equal peptides, mostly because they are the closest paralogs that have been found and purified together. ${ }^{16}$ They are commonly referred as MT-1/MT-2. However, the availability of transcriptomic and proteomic techniques highlights differences in MT-1 and MT-2 that cannot be ignored, suggesting some degree of functional differentiation between these isoforms both within the same species as well as between humans and rabbits.

Amino acid sequences of rabbit MT isoforms show differences when compared to the sequences of human MTs (Fig. 1). Human MTs are controlled by 17 genes located on chromosome 16, 10 of which are functional. To date, 10 subisoforms of human liver MT-1/2 have been reported (MT-1A, MT-1B, MT-1E, MT-1F, MT-1G, MT-1H, MT-1M, MT-1L, MT-1X, and MT-2A) ${ }^{2}$; their amino acid sequences are shown in Fig. 1A. Subisoform MT-1G contains 62 amino acids, whereas the others display a structure of 61 amino acids.

Rabbit MT genes are located on chromosome 5. Six subisoforms of rabbit liver MT-1/2 have been reported so far (MT-1A, MT-2A, MT-2B, MT-2C, MT-2D, and MT-2E) ${ }^{17}$; their amino acid sequences are presented in Fig. $1 \mathrm{~B}$. Subisoforms MT-2A and MT-2C contain 62 amino acids, whereas the others display a structure of 61 amino acids. The reasons for the high diversity of MT isoforms and their specific functional roles are still unclear. The potential biological significance of each isoform should not be neglected but the analytical techniques available cannot easily separate them. ${ }^{18}$

By ion-exchange chromatography, human MTs were separated into 2 charge-separable isoforms, designated as MT fractions 1 and $2 .{ }^{17}$ However, MT fractions from rabbit liver obtained with the same ion-exchange chromatography cannot be separated into MT-1 and MT-2 subfractions. This conventional nomenclature division based on charge differences refers to the absence (MT-1) or presence (MT-2) of an aspartic acid residue at position 11 or 12 (10 or 11$)$ of the sequence (Fig. 1) and it is not based on their global charge. ${ }^{19}$

Amino acid sequences of 6 rabbit MT isoforms show some differences compared to the sequences of human MTs (Fig. 1). Owing to the insertion of an additional amino acid at position 10, MT-2A and MT-2C sequences have a total chain length of 62 amino acids. MT-1G, having the same length and the same insertion, has also been identified in human liver. The presence of differential expression of MT-1G1 (with an additional alanine) and MT-1G2 (without additional alanine) suggests tissue- and cell-specific alternative splicing for the MT-1G isoform. ${ }^{20}$ The insertion of the alanine residue at position 10 of the sequence relates to the location of the connection of exons 1 and 2 of the rabbit 


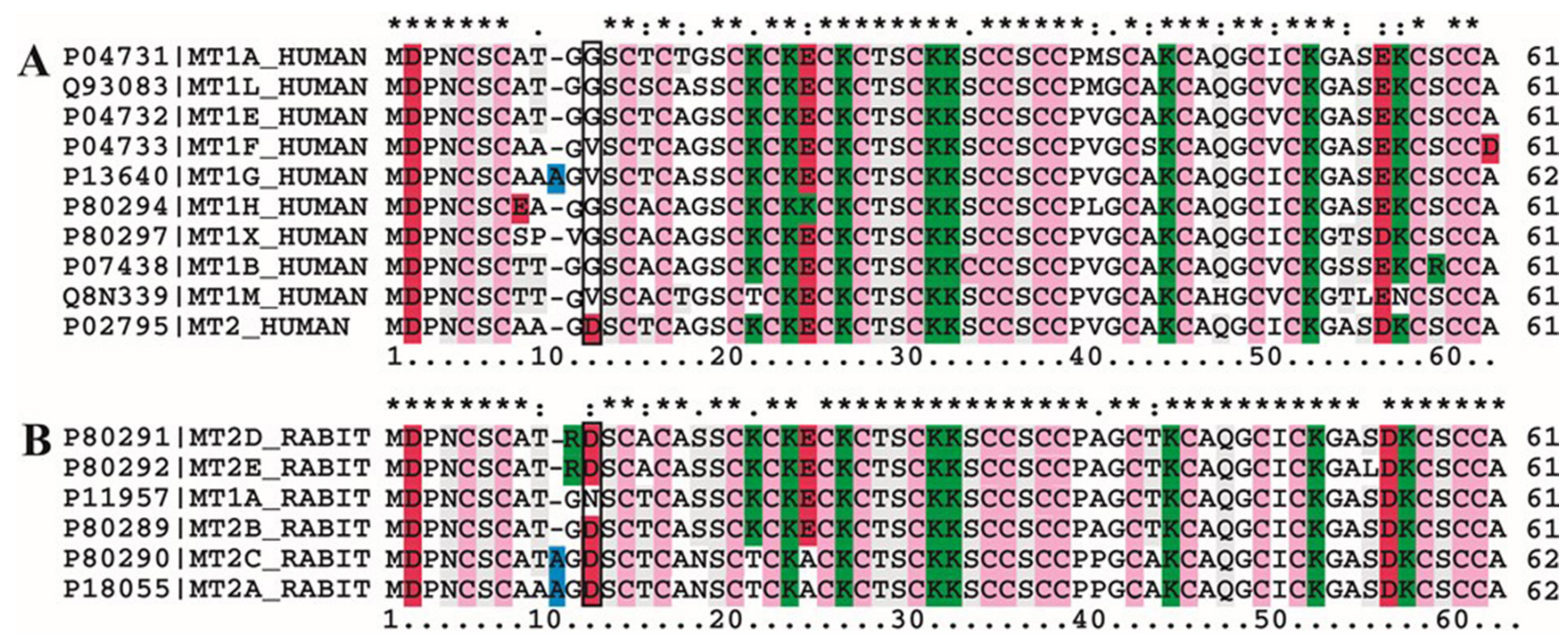

Fig. 1. Comparison of human and rabbit metallothionein (MT) isoforms 1 and 2 sequences (MT-1 and MT-2). Sequence comparison of MT-1/2 from human (MT-1A, MT-1B, MT-1E, MT-1F, MT-1G, MT-1H, MT-1M, MT-1L, and MT-1X, MT-2A) and from rabbit (MT-1A, MT-2A, MT-2B, MT-2C, MT-2D, and MT-2E) was performed using ClustulX program. ${ }^{37}$ The sequences were derived from the database SWISS PROT (http://www.expasy.org/sprot/)

* indicates positions which have a single, fully conserved residue; : indicates conservation between groups of strongly similar properties - scoring > 0.5; indicates conservation between groups of weakly similar properties - scoring $\leq 0.5$. The red color indicates negative amino acid residues; the color green indicates positive amino acid residues; the pink color indicates cysteine residues; the blue color marks alanine - an additional amino acid at position 10; substitution of an aspartic acid residue at position $11 / 12$ is indicated by the frame.

MT-2A and 2C gene and human MT-1G gene. The presence of the additional alanine does not seem to influence neither the tertiary structure nor the metal-binding properties of the protein. ${ }^{17,21}$ The biological consequence of that insertion remains undefined but its presence in more than 1 species suggests a common mechanism leading to the same phenotype.

In rabbit MT-2D and MT-2E, there is a substitution of arginine $(\mathrm{R})$ residue in position $10 / 11$ (Fig. 1B). The additional positive charge may be involved in compensating for the net negative charge of the 3-metal-cluster and thus can increase the stability of the $\beta$-cluster. ${ }^{17}$

Even minor changes in the sequence of MT may induce structural changes that can alter the metal-binding properties or stability of the 3-metal-cluster. The flexibility of the polypeptide chain as well as the charge environment of the coordinating sulfur atoms may greatly influence the feasibility and stability of the metal-thiolate bonds, and as a result, of the final metal-MT complex.

Aras et al. noticed a conserved PKC phosphorylation site at serine-32 (S32) of MT, which was important in modulating $\mathrm{Zn}^{2+}$-regulated gene expression. ${ }^{22}$ It is present in all rabbit and human MT isoforms shown in Fig. 1, except human MT-1B. The presence of cysteine in place of serine can change the tertiary structure of the protein as well as change the function of the protein dependent on phosphorylation.

Different expression patterns of MT subisoforms are observed in various types of human malignancies; this fact can be useful in the diagnosis and therapy of tumors. ${ }^{3}$ The exact biological significance of the differences in amino acid sequences in human and rabbit MTs still remains unclear.

\section{Detection of metallothioneins using capillary gel electrophoresis}

Capillary electrophoresis is often used to analyze and separate MT isoforms from different organisms and tissues. ${ }^{11,23}$ It can also be used for monitoring MT concentration in biological samples. ${ }^{23}$ Křížková et al. were the first to propose a chip CE technique for the analysis of MT, i.e., of the effect of oxidation on the structure of MT and its ability to form aggregates and polymers. ${ }^{24}$ Unfortunately, due to the presence of system peaks, an accurate analysis of the MT monomer is difficult. ${ }^{12,25}$

In this study, changes in the range of 5-8 kDa corresponding to the MT monomer were observed for both human and rabbit MTs. However, the monomers co-migrated with a system peak, which made it difficult to measure their correct size. In Fig. 2, the peak obtained for rabbit MT shifted to a bigger mass $(8.2 \mathrm{kDa})$ in comparison to human MTs (6.0 and 7.7 kDa for human MT-1, and 5.3 and 6.1 $\mathrm{kDa}$ for human MT-2). An additional peak was observed at about 23-24 min, which may indicate the presence of 13.4 and $14.0 \mathrm{kDa}$ oligomers. Peaks corresponding to MT dimers were observed both for human and rabbit MTs.

\section{Mass spectrometry analysis}

In the majority of MALDI-MS studies, DHB and HCCA were the constituents of the matrix used for the appropriate determination of MTs. ${ }^{26}$ In our study, utilization of both matrixes resulted in different types of crystals. While HCCA produced uniform crystals, DHB produced 


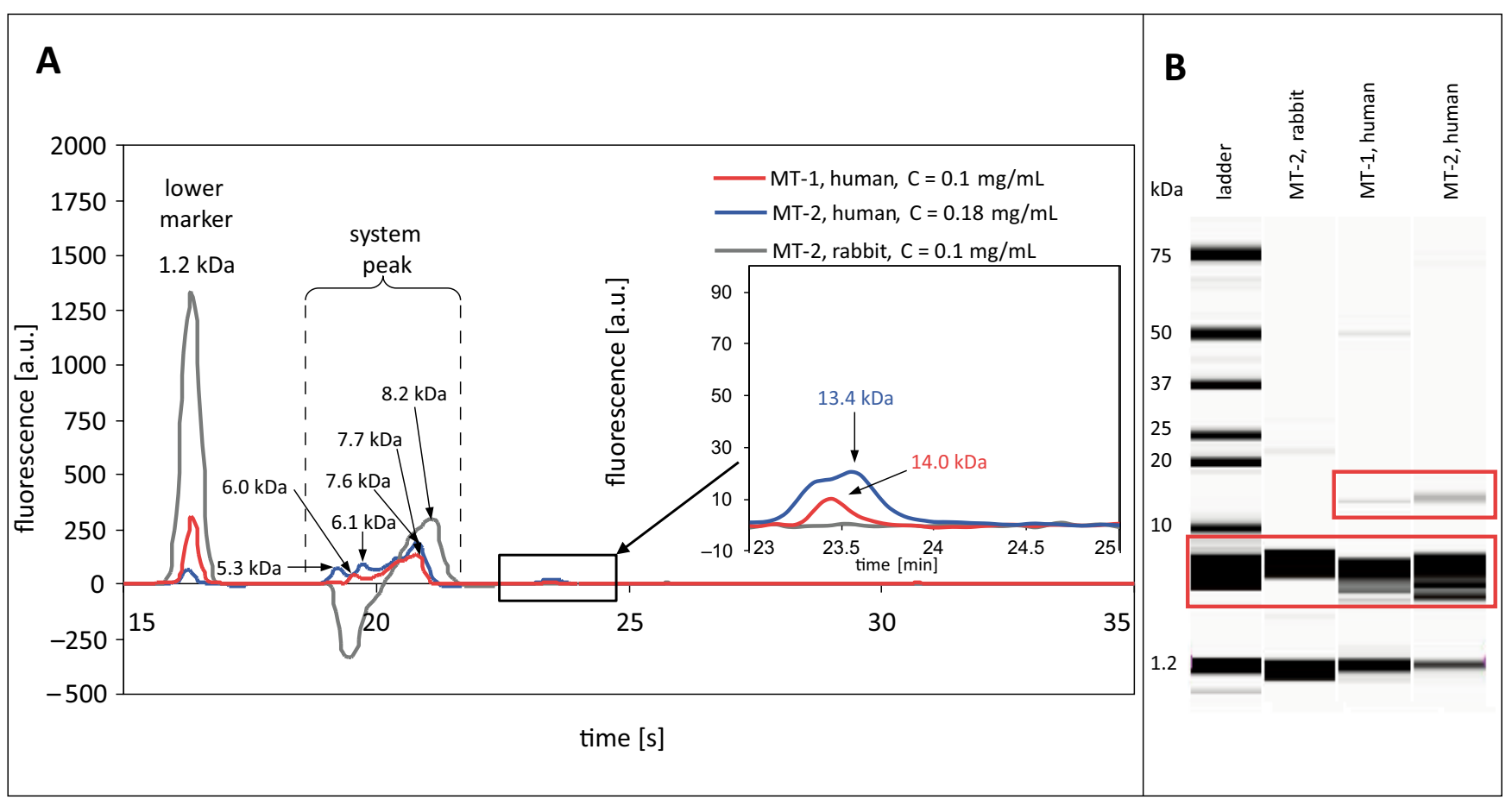

Fig. 2. Metallothionein (MT) separation using chip capillary gel electrophoresis (CE). Electropherograms of: A) in red, MT-1 from human liver; in blue, MT-2 from human liver; and in gray, MT-2 from rabbit liver are shown; B) chip CE virtual gel output of Pro260 ladder, MT-2 from rabbit liver, MT-1 and MT-2 from human liver

heterogeneous and robust crystals (Fig. 3A). Furthermore, DHB exhibited a significant rabbit MT-2 concentrationdependent increase in observed signal intensity (expressed in a.u.), higher when compared to that of HCCA (Fig. 3B). The main observed signal for rabbit MT-2 was $6211.07 \mathrm{~m} / \mathrm{z}$. Moreover, it was estimated that the isolated MT-2 was highly pure, because each spectrum showed only 1 peak of high intensity for the relevant protein. This peak showed a mass similar to rabbit MT-2D $(6215.40 \mathrm{~m} / \mathrm{z}) .{ }^{27}$ The occurrence of the MT-2D form has been also observed in MT-2 from Sigma lots ${ }^{27,28}$ and in MT-1 sample, as a remaining contaminant. ${ }^{28}$ The size of 12342.11 Da corresponds to the MT-2 dimer (Fig. 3C inset).

The DHB was chosen as the most appropriate matrix in terms of sensitivity and reproducibility for human MTs; at given conditions $(20 \mathrm{mg} / \mathrm{mL}$ of $\mathrm{DHB}$ in $30 \% \mathrm{ACN}$ and $1 \%$ TFA), homogeneous spots applicable for automated MALDI-TOF-MS were formed. Further, to verify the proper isolation of MT-1 and MT-2 from humans, we analyzed their mass distribution. The main observed signals for human MTs (hMTs) shown in Fig. 4 were quasimolecular ions assigned as follows: [hMT-1] ${ }^{+}(6145.23 \mathrm{~m} / \mathrm{z}, 6176.46 \mathrm{~m} / \mathrm{z}$ and $6220.12 \mathrm{~m} / \mathrm{z}$ ) (Fig. 4A), and [hMT-2]+ $(6066.24 \mathrm{~m} / \mathrm{z})$ (Fig. 4B). The sizes of $12523.89 \mathrm{Da}$ and $12329.89 \mathrm{Da}$ correspond to MT-1 and MT-2 dimers. The mass spectra of the human MT-1 sample yielded a main peak, the most intense at 6176.46 Da. Peaks observed in the spectra of these MT-1 samples can be attributed to potential subisoforms: MT-1A $(6145.23 \mathrm{~m} / \mathrm{z})$, MT-1G $(6176.46 \mathrm{~m} / \mathrm{z})$ and MT-1G+Cd $(6240.12 \mathrm{~m} / \mathrm{z}){ }^{29,30}$ The theoretical mass of human MT-2A is $6042.16 \mathrm{~m} / \mathrm{z}$ and our results showed main signals at $6066.24 \mathrm{~m} / \mathrm{z}$, corresponding to [hMT-2A+Na] $]^{+}$.

The exposure of MTs to acidic $\mathrm{pH}$ values causes metal depletion and unfolding of the protein structure, generating apothioneins or metal-free forms. Using these matrixes caused the coordinated metals of the proteins to be removed, and the peaks obtained should be apothioneins, i.e., subisoforms without metal content.

Metallothionein dimers were first detected as transient intermediate species during interprotein metal exchange, and longer-lived dimers have been isolated that were stabilized by intermolecular disulfide bonds. ${ }^{31}$ No transient intermediate dimers could be detected with mass spectrometry. However, a stable dimeric complex of MTs has been detected in this work by MALDI-TOF-MS and chip $\mathrm{CE}$, and it appears to be stabilized by disulfide bridging. Metallothioneins can dimerize through the oxidation of their cysteinyl residues to form an interprotein CysCys dithiol bridge; the experimental mass would decrease by $2 \mathrm{Da}$ for every oxidative thiol bridge formation event.

\section{Metallothionein analysis using the Brdicka reaction}

Metallothionein exhibits significant electrochemical activity owing to its high content of sulfhydryl (SH) moieties. The method of determining proteins which contain -SH groups in an ammonia-buffered cobalt (III) solution was first described by Brdička. ${ }^{32}$ Brdicka reaction is one of the mostoften-used electrochemical methods for MT determination 

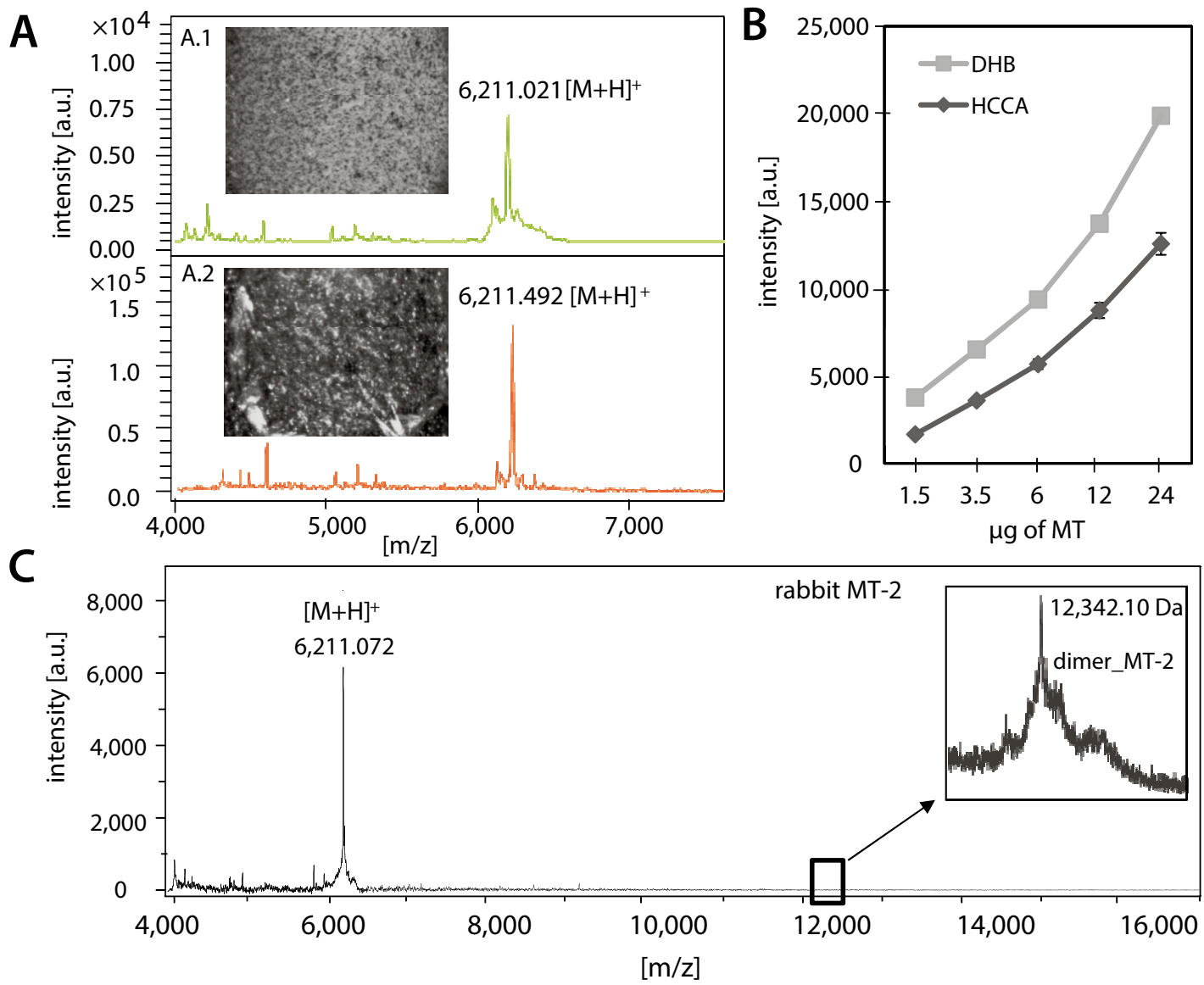

Fig. 3. Mass spectrum of rabbit metallothionein (MT). A) Spectrum of MT-2 from rabbit liver measured by matrix-assisted laser desorption and ionization time-of-flight mass spectrometry (MALDI-TOF-MS) with (A.1) a-cyano-4-hydroxycinnamic (HCCA) and (A.2) 2,5-dihydroxybenzoic acid (DHB) matrix. B) Graphs of signal intensity of different concentrations of rabbit MT-2 in DHB and HCCA matrices. 1-2 $\mu \mathrm{L}$ of sample/matrix mixture (1:1) deposited on MALDI plate and dried at room temperature (dried-droplet method). Analysis by a MALDI-TOF-MS mass spectrometer was performed in linear and reflector mode. Dimerization of MT analyzed by MALDI-TOF-MS is shown in the inset of Fig. C) Spectrum of rabbit MT-2 measured by MALDI-TOF-MS with the DHB matrix in linear positive mode in a mass range of 4-17 kDa prepared in TA30 with repetition rate of $2000 \mathrm{~Hz}$

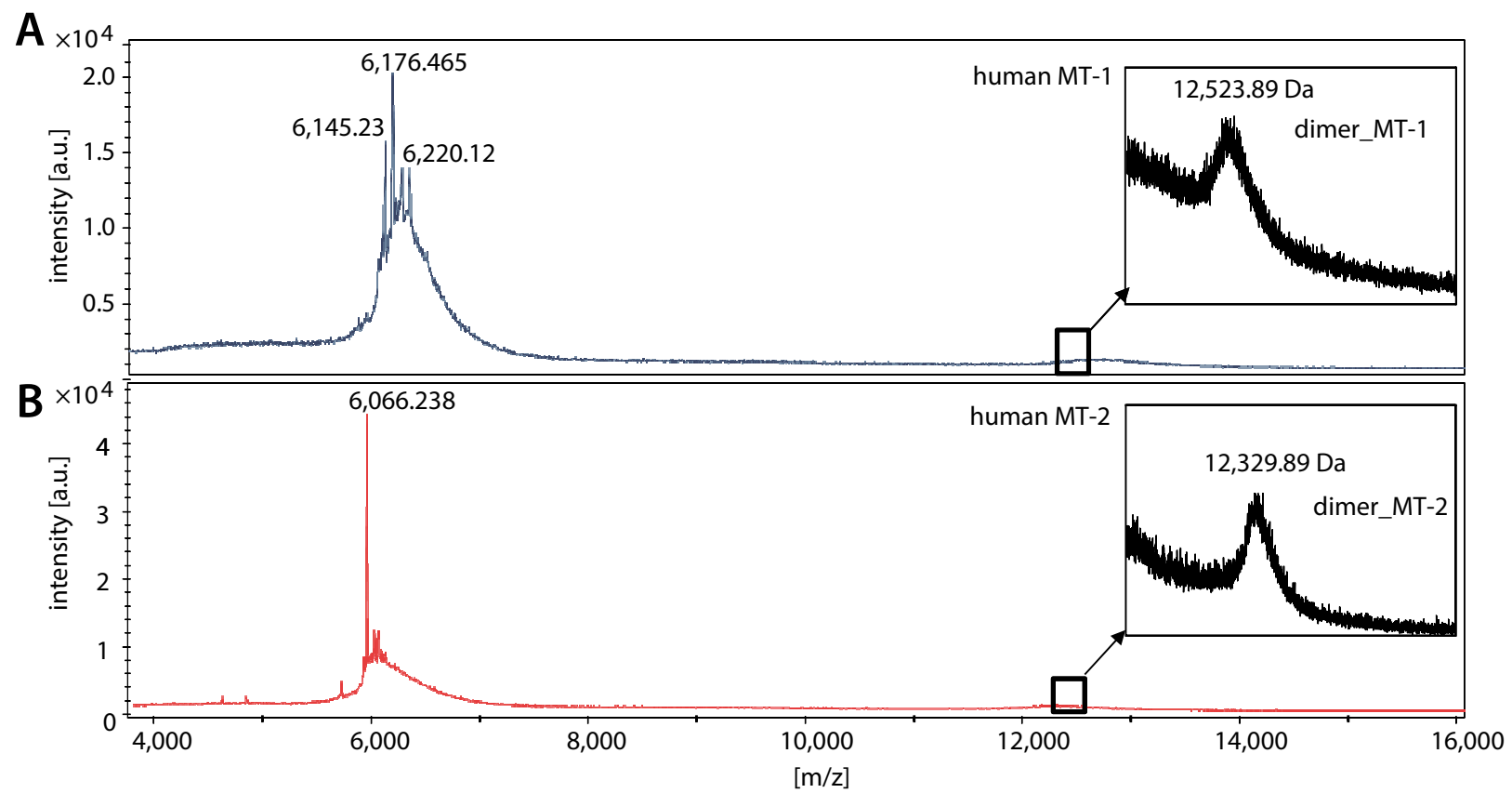

Fig. 4. Mass spectrum of human metallothionein (MT). Spectrum of MT-1 (A) and MT-2 (B) from human measured by matrix-assisted laser desorption and ionization time-of-flight mass spectrometry (MALDI-TOF-MS) with the 2,5-dihydroxybenzoic acid (DHB) matrix in linear positive mode in a mass range of 4-16 kDa prepared in TA30 with repetition rate of $2000 \mathrm{~Hz}$ 
A

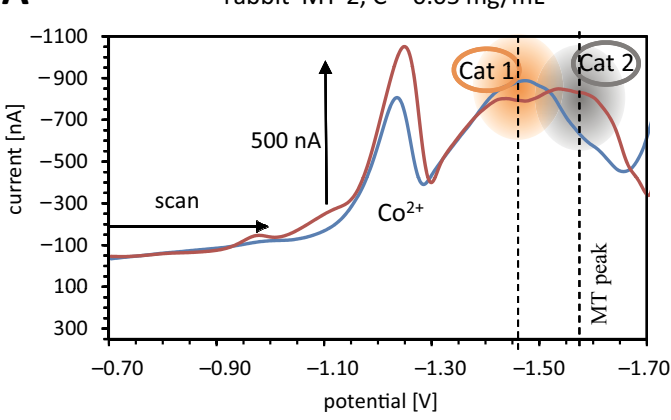

B

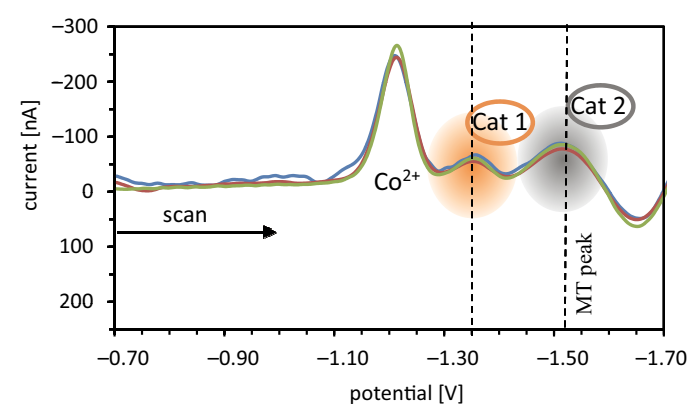

C human $\mathrm{MT}-2, \mathrm{C}=0.05 \mathrm{mg} / \mathrm{mL}$

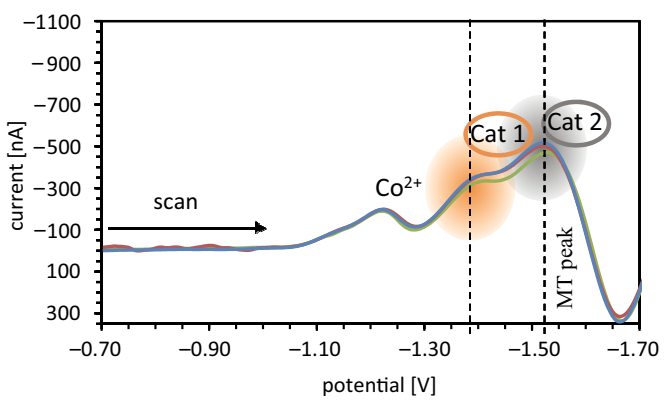

D

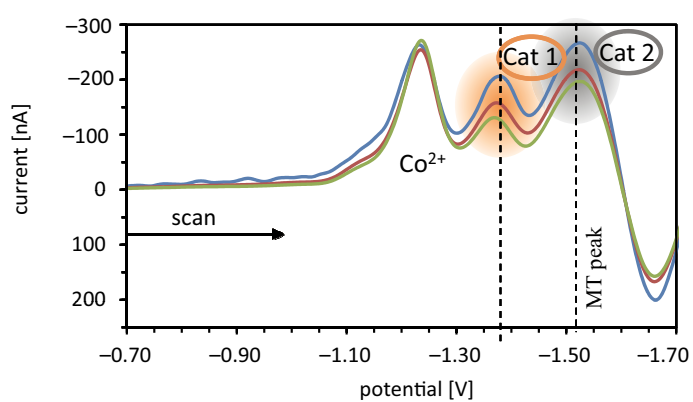

Fig. 5. Metallothioneins (MTs) from rabbit and human liver analysis using the Brdicka reaction. Different concentrations of MT were used: $0.025 \mathrm{mg} / \mathrm{mL}$ and $0.05 \mathrm{mg} / \mathrm{mL}$.

in biological samples. ${ }^{33}$ It is based on the reduction of hydrogen at the mercury electrode catalyzed by the $-\mathrm{SH}$ groups in the ammonia-buffered cobalt(III) solution. ${ }^{34}$

The Brdicka reaction has potential for monitoring the differences of various MT isoforms, both rabbit and human. ${ }^{26}$ During MT analysis, changes in Cat 2 signal $(-1.55 \mathrm{~V}$, which represents the current response of the MT complex with components of the Brdicka electrolyte), $\mathrm{Co}^{2+}(-1.25 \mathrm{~V})$ and Cat1 $(-1.40 \mathrm{~V})$ corresponding to hydrogen evolution from the supporting electrolyte catalyzed by the MT were observed..$^{35}$ The presence of the Cat 2 peak is closely connected with the quantification of $-\mathrm{SH}$ groups occurring in the Brdicka solution, ${ }^{36}$ thus corresponding to the MT level. The voltammograms of human and rabbit MT are shown in Fig. 5. It can be observed that the character of the mentioned MT signals changes with different MT concentration both for rabbit and human MT (Fig. 5). Concentrations of MT 0.025 and $0.05 \mathrm{mg} / \mathrm{mL}$ were analyzed.

With a lower concentration of rabbit $\mathrm{MT}$, the $\mathrm{Co}^{2+}$ signal decreases and shifts to a less negative potential, whereas it slightly increases with a decreasing concentration of human MT (Fig. 5). Cat1 and Cat2 signals are more exposed in a lower concentration of MTs. With a lower rabbit MT concentration, Cat 1 and Cat 2 signals decreased and shifted to a more positive potential. These signals in the lower concentration of human MT had a negligible potential shift. The character of the mentioned MT signals changed with MTs concentrations. A lower concentration of MTs from both organisms coincided with a decrease in the Cat2 signal. Lower, but more distinct signals were observed for human and rabbit MTs when a lower concentration was used $(0.025 \mathrm{mg} / \mathrm{mL})$. The same concentration of human MT gave higher Cat2 peak than rabbit MT. Metallothioneins isolated in both organisms behave differentially despite the same number of $-\mathrm{SH}$ groups.

\section{Conclusions}

The structure of MTs is constantly being studied due to their dynamic nature, lack of secondary structural features when metals are not present, and the absence of spectroscopically active aromatic residues. Thus, different methods have been used for the analysis of human MT-1 and MT-2 isoforms and rabbit MT-2 isoforms.

Both in rabbit and human MTs, mainly monomers were detected with chip CE and MALDI-TOF-MS. Additionally, distinctly lower signals from the dimers were observed. When MALDI-TOF-MS is employed, the main observed signal for rabbit MT-2 corresponds to the mass of MT-2D. In human, MT-1A, MT-1G, MT-1G+Cd and MT-2A were detected. Metallothionein subisoforms, except in the case of human MT-1G+Cd, have been identified without metals, which can be due to the use of acidic matrixes that cause a release of less bound metals.

In the Brdicka reaction, signals are different for MTs isolated from rabbit and human liver, meaning that they behave differentially in electrochemistry despite the same 
number of - $\mathrm{SH}$ groups. This may be due to different availability of MT cysteinyl groups in different organisms.

These methods used together allow for the identification and characterization of MT and give complementary information about MT forms. Furthermore, MALDI-TOF-MS is capable of directly resolving more than just the main MT isoforms in the sample, which makes it the best of these techniques to identify isoforms of MT.

\section{References}

1. Osobová M, Urban V, Jedelský PL, et al. Three metallothionein isoforms and sequestration of intracellular silver in the hyperaccumulator Amanita strobiliformis. New Phytol. 2011;190(4):916-926.

2. Zalewska M, Trefon J, Milnerowicz H. The role of metallothionein interactions with other proteins. Proteomics. 2014;14(11):1343-1356.

3. Křížková S, Kepinska M, Emri G, et al. An insight into the complex roles of metallothioneins in malignant diseases with emphasis on (sub)isoforms/isoforms and epigenetics phenomena. Pharmacol Ther. 2017; 183:90-117.

4. Torreggiani A, Chatgilialoglu C, Ferreri C, Melchiorre M, Atrian S, Capdevila M. Non-enzymatic modifications in metallothioneins connected to lipid membrane damages: Structural and biomimetic studies under reductive radical stress. J Proteomics. 2013;92:204-215.

5. Macirella R, Brunelli E. Morphofunctional alterations in zebrafish (Danio rerio) gills after exposure to mercury chloride. Int J Mol Sci. 2017;18(4):824.

6. Sanz-Nebot V, Andón B, Barbosa J. Characterization of metallothionein isoforms from rabbit liver by liquid chromatography coupled to electrospray mass spectrometry. J Chromatogr B Analyt Technol Biomed Life Sci. 2003;796(2):379-393.

7. Ruttkay-Nedecky B, Nejdl L, Gumulec J, et al. The role of metallothionein in oxidative stress. Int J Mol Sci. 2013;14(3):6044-6066.

8. Artells E, Palacios O, Capdevila M, Atrian S. In vivo-folded metalmetallothionein 3 complexes reveal the $\mathrm{Cu}$-thionein rather than $\mathrm{Zn}$ thionein character of this brain-specific mammalian metallothionein. FEBS J. 2014;281(6):1659-1678.

9. Thirumoorthy N, Shyam Sunder A, Manisenthil Kumar K, Senthil Kumar M, Ganesh G, Chatterjee M. A review of metallothionein isoforms and their role in pathophysiology. World J Surg Oncol. 2011;9:54.

10. Milnerowicz H, Bizoń A. Determination of metallothionein in biological fluids using enzyme-linked immunoassay with commercial antibody. Acta Biochim Pol. 2010;57(1):99-104.

11. Zalewska M, Bizoń A, Milnerowicz H. Comparison of capillary electrophoretic techniques for analysis and characterization of metallothioneins. J Sep Sci. 2011;34(21):3061-3069.

12. Guszpit E, Kř́žková S, Kepinska M, et al. Fluorescence-tagged metallothionein with CdTe quantum dots analyzed by the chip-CE technique. J Nanopart Res. 2015;17(11):423.

13. Guszpit E, Krejčová L, Kř́žková S, et al. Kinetic analysis of human metallothionein and $\mathrm{CdTe}$ quantum dot complexes using fluorescence and voltammetry techniques. Colloids Surf B Biointerfaces. 2017; 160:381-389.

14. Dabrio M, Virtanen V, Bordin G, Rodriguez AR. Contribution to the study of complexing properties of Zn-metallothioneins by CZE-DAD. Talanta. 2000;53(3):587-598.

15. Mounicou S, Ouerdane L, L'Azou B, et al. Identification of metallothionein subisoforms in HPLC using accurate mass and online sequencing by electrospray hybrid linear ion trap-orbital ion trap mass spectrometry. Anal Chem. 2010;82(16):6947-6957.

16. Artells E, Palacios Ò, Capdevila M, Atrian S. Mammalian MT1 and MT2 metallothioneins differ in their metal binding abilities. Metallomics. 2013;5(10):1397-1410.

17. Hunziker $P E$, Kaur $P$, Wan $M$, Känzig A. Primary structures of seven metallothioneins from rabbit tissue. Biochem J. 1995;306(Pt 1): 265-270.
18. Beattie JH. Strategies for the qualitative and quantitative analysis of metallothionein isoforms by capillary electrophoresis. Talanta. 1998;46(2):255-270.

19. Kojima Y, Hunziker PE. Amino acid analysis of metallothionein. Methods Enzymol. 1991;205:419-421.

20. Mehus AA, Muhonen WW, Garrett SH, Somji S, Sens DA, Shabb JB. Quantitation of human metallothionein isoforms: A family of small, highly conserved, cysteine-rich proteins. Mol Cell Proteomics. 2014; 13(4):1020-1033.

21. Braun W, Vasák M, Robbins AH, et al. Comparison of the NMR solution structure and the $x$-ray crystal structure of rat metallothionein-2. Proc Natl Acad Sci U S A. 1992;89(21):10124-10128.

22. Aras MA, Hara H, Hartnett KA, Kandler K, Aizenman E. Protein kinase $C$ regulation of neuronal zinc signaling mediates survival during preconditioning. J Neurochem. 2009;110(1):106-117.

23. Ryvolova M, Hynek D, Skutkova H, Adam V, Provaznik I, Kizek R. Structural changes in metallothionein isoforms revealed by capillary electrophoresis and Brdicka reaction. Electrophoresis. 2012;33(2):270-279.

24. Kř́žzová S, Adam V, Kizek R. Study of metallothionein oxidation by using of chip CE. Electrophoresis. 2009;30(23):4029-4033.

25. Kř́žková S, Masařík M, Eckschlager T, Adam V, Kizek R. Effects of redox conditions and zinc(II) ions on metallothionein aggregation revealed by chip capillary electrophoresis. J Chromatogr A. 2010;1217(51): 7966-7971.

26. Merlos Rodrigo MA, Molina-López J, Jimenez Jimenez AM, et al. The application of curve fitting on the voltammograms of various isoforms of metallothioneins-metal complexes. Int J Mol Sci. 2017; 18(3):610.

27. van Vyncht $G$, Bordin G, Rodriguez AR. Rabbit liver metallothionein subisoform characterization using liquid chromatography hyphenated to diode array detection and electrospray ionization mass spectrometry. Chromatographia. 2000;52(11-12):745-752.

28. Chassaigne H, Lobinski R. Polymorphism and identification of metallothionein isoforms by reversed-phase HPLC with on-line ion-spray mass spectrometric detection. Anal Chem. 1998;70(13):2536-2543.

29. Tomalová I, Foltynová P, Kanický V, Preisler J. MALDI MS and ICP MS detection of a single CE separation record: A tool for metalloproteomics. Anal Chem. 2014;86(1):647-654.

30. Irvine GW, Pinter TBJ, Stillman MJ. Defining the metal binding pathways of human metallothionein 1a: Balancing zinc availability and cadmium seclusion. Metallomics. 2016;8(1):71-81.

31. Irvine GW, Heinlein L, Renaud JB, Sumarah MW, Stillman MJ. Formation of oxidative and non-oxidative dimers in metallothioneins: Implications for charge-state analysis for structural determination. Rapid Commun Mass Spectrom. 2017;31(24):2118-2124.

32. Brdička R. Polarographic studies with the dropping mercury electrode. Part IV. The influence of circuit resistance on maxima of current-voltage curves. Collect Czechoslov Chem Commun. 1936;8: 419-433.

33. Raspor B, Paić M, Erk M. Analysis of metallothioneins by the modified Brdicka procedure. Talanta. 2001;55(1):109-115.

34. Kř́žková S, Blahova P, Nakielna J, et al. Comparison of metallothionein detection by using Brdicka reaction and enzyme-linked immunosorbent assay employing chicken yolk antibodies. Electroanalysis. 2009;21(23):2575-2583.

35. Kruseova J, Hynek D, Adam V, et al. Serum metallothioneins in childhood tumors: A potential prognostic marker. Int J Mol Sci. 2013;14(6): 12170-12185.

36. Adam V, Chudobova D, Tmejova K, et al. An effect of cadmium and lead ions on Escherichia coli with the cloned gene for metallothionein (MT-3) revealed by electrochemistry. Electrochimica Acta. 2014;140: $11-19$.

37. Thompson JD, Gibson TJ, Plewniak F, Jeanmougin F, Higgins DG. The CLUSTAL_X windows interface: Flexible strategies for multiple sequence alignment aided by quality analysis tools. Nucleic Acids Res. 1997;25(24):4876-4882. 